\title{
UMA ANÁLISE DA PERCEPÇÃO DOS ESTUDANTES DE ENGENHARIA DE PRODUÇÃO QUANTO À QUALIDADE DAS INSTITUIÇÕES DE ENSINO SUPERIOR EM 2014 E 2017
}

\begin{abstract}
Maurício Valdson da Silva Barreira - mauricio.barreira@fapce.edu.br Centro Universitário Paraíso - Departamento de Engenharia de Produção Endereço: Rua Ecy Brito Mariano, 395 - Bairro: Parque Grangeiro CEP: 63106-032 - Crato - Ceará

Leonardo de Sousa Silva - leonardo.sousa@fapce.edu.br Centro Universitário Paraíso - Departamento de Engenharia de Produção Endereço: Rua Antônio Cândido Sobrinho, $n^{\circ}$ 39, casa 6 - Bairro: Aeroporto CEP: 63020-740 - Juazeiro do Norte - Ceará
\end{abstract}

Thiago Mársis Braga Diniz - thiago.diniz@ifce.edu.br Instituição Federal de Educação, Ciência e Tecnologia do Ceará - IFCE Endereço: Rua Manoel Gouveia Silva, 523 - Bairro: Aeroporto CEP: 63021-220 - Juazeiro do Norte - Ceará

Francisco Eduardo de Lima Nascimento - fel.nascimento@unesp.br Universidade Estadual Paulista - UNESP - Guaratinguetá Endereço: Rua Sr. Amaro, 190 - Bairro: Bugi CEP: 63501-045 - Iguatu-Ceará

Resumo: O número de cursos de engenharia de produção como também de seus ingressantes vêm registrando um aumento frequente. Nesse sentido, esse estudo analisou o impacto de ações das Instituições de Ensino Superior representadas por variáveis qualitativas. Para isso, utilizou-se como indicador a percepção dos estudantes dos cursos de Engenharia de Produção quanto à qualidade das Instituições de Ensino Superior nos anos de 2014 e 2017, analisando as respostas de 42 questões qualitativas pertencentes ao Questionário do Estudante. Tais questões refletem perguntas relacionadas aos aspectos do desempenho das instituições. A partir da análise dos resultados, verificou-se que não existe uma diferença significativa na percepção geral média dos estudantes. $O$ estudo demonstrou a relevância da análise estatística sobre os resultados do Enade, e direciona que novas variáveis que possam influenciar nesses resultados sejam analisadas em trabalhos futuros.

Palavras-chave: Engenharia de produção. Enade. Questionário do estudante. Percepção do estudante.

\section{INTRODUÇÃO}

A Engenharia de Produção tem conquistado um espaço cada vez maior nas instituições de ensino do Brasil e a procura por cursos de graduação em Engenharia de Produção vem se intensificando juntamente com o aprimoramento dos sistemas de produção. Diante desse contexto é de suma importância para a empresa a contratação de um profissional apto a 
gerenciar os sistemas produtivos de modo eficiente e eficaz, utilizando os recursos disponíveis de maneira otimizada.

O último Censo da Educação do Ensino Superior concluído em 2019 (INEP, 2019), continua a mostrar o crescente aumento de Instituições de Ensino Superior (IES) no país. A oferta do curso de Engenharia de Produção Plena também tem aumentado conforme os dados extraídos das sinopses estatísticas da base de dados do INEP (2020). Para Melo (2017) vem acontecendo um grande movimento no país de migração das Engenharias de Produção com determinadas habilitações (ênfases) para a Engenharia de Produção Plena, por esta última ser mais representativa entre as modalidades. Tal fato pode ser evidenciado na visão de muitos autores. Segundo Ribeiro et al. (2019), "os engenheiros são peças fundamentais para a sobrevivência destas empresas, pois sua formação é pautada em solução de problemas, análise de custos, tecnologia e conhecimentos técnicos". Para Cardoso (2007) é uma graduação de caráter sistêmico, abrangendo conhecimentos de várias áreas como tecnologia, administração, ciências econômicas, psicologia, entre outras, o tornando um profissional de conhecimento e habilidades amplas. Dessa forma, a exigência cada vez maior por profissionais altamente capacitados, tem aumentado a quantidade de instituições oferecendo essa modalidade de curso (CONRADSEN e LYSTLUND, 2003).

Porém, esse crescimento na oferta de cursos de Engenharia de Produção, também acirra a disputa entre as IES, fazendo com que muitas adotem estratégias de menor preço, flexibilidade e facilidade no ingresso ao nível superior, a fim de captar o maior número de alunos possível.

Em meio a esta situação, é importante verificar se tal fato, não tem afetado a qualidade do processo formativo nestas instituições. Segundo Camelo e Marcião (2019), essa expansão tem causado problemas relacionados da ausência de profissionalização da gestão e pouco comprometimento em relação à qualidade do processo educacional. Estes mesmos autores afirmam ainda, que as IES que não aprimorarem seus processos de gestão, ensino e aprendizagem fatalmente irão fracassar.

Nesse contexto, esse estudo avaliou a percepção dos estudantes dos cursos de Engenharia de Produção quanto à qualidade das Instituições de Ensino Superior nos anos de 2014 e 2017, tomando por base os microdados do INEP/ENADE dos referidos anos, analisando as respostas de 42 questões qualitativas pertencentes ao Questionário do Estudante, que avaliam aspectos das IES's divididos em quatro categorias: i) processo ensino-aprendizagem; ii) gestão educacional; iii) ação docente e iv) estrutura física.

As questões foram quantificadas através de um método que relaciona os percentuais de cada resposta com um valor de uma escala Likert, gerando uma pontuação individual para cada uma. A partir disso, foi realizada uma análise comparando os valores individuais de cada questão, o valor médio de cada categoria e o valor médio geral entre os anos.

Ao final dos estudos, foi observado nos resultados que não existe diferença significativa na percepção geral média dos estudantes entre os anos de 2014 e 2017. A diferença entre as categorias apresentou redução na pontuação média para Processo Ensino-Aprendizagem e Ação Docente e aumento para Gestão Educacional e Estrutura Física.

\section{REFERENCIAL TEÓRICO}

Segundo Queiroz et al. (2011), o surgimento e a consolidação da Engenharia de Produção no país estão diretamente ligados ao crescimento econômico brasileiro e ao desenvolvimento da indústria brasileira. Em 1970 na USP em São Carlos e em 1971 na Universidade Federal do Rio de Janeiro (UFRJ) surgiram os cursos de Engenharia de Produção como primeira modalidade autônoma com formação plena (BITTENCOURT et al., 2010). De acordo com 
Oliveira et al. (2013), a partir de 1996 com uma grande expansão dos cursos superiores, no país já havia 35 cursos de Engenharia de Produção, sendo que até o ano 2000 este número quase que triplicou. Em 2013, dentre os 3.050 cursos de Engenharia existentes no país, cerca de 550 eram de Engenharia de Produção, de acordo com dados tabulados a partir do Portal EMEC. Em 2017 havia um total de 805 cursos, e 2018 atingindo o patamar de 878 cursos.

Apesar de toda a expansão dos cursos de Engenharia de Produção no Brasil e da proposta trazida na formação desse profissional vista até o momento, não se tem visto um crescimento proporcional qualitativo (OLIVEIRA et al., 2013).

O ENADE é componente curricular obrigatório dos cursos de graduação do país e tem como objetivo aferir o desempenho dos estudantes em relação aos conteúdos programáticos previstos nas diretrizes curriculares do curso de graduação.

Conforme consta em Oliveira e Fernandes (2015), o ENADE foi criado pela Lei $\mathrm{N}^{\circ}$ 10.861/2004 que instituiu o Sistema Nacional de Avaliação da Educação Superior (SINAES). O órgão responsável pela avaliação do ensino superior brasileiro é o INEP, uma autarquia federal vinculada ao Ministério da Educação.

De acordo com a legislação, devem ser inscritos no exame os estudantes dos cursos de graduação, ingressantes e concluintes dos cursos avaliados, bem como os estudantes irregulares. A seguir tem-se uma descrição dos estudantes aptos para realizar a inscrição:

- Inscrição dos Estudantes Irregulares: Estudantes habilitados no Enade de anos anteriores que, por qualquer razão, permaneceram em situação irregular e não foram beneficiados por dispensa oficial concedida pelo MEC. A IES deverá inscrevê-los para regularização de sua situação junto ao Enade, no entanto, esses Estudantes não realizarão o Exame.

- Inscrição dos Estudantes Ingressantes: Estudantes que tenham iniciado o respectivo curso no ano de realização do Enade, devidamente matriculados, e que tenham até vinte e cinco por cento da carga horária mínima do currículo do curso cumprida até o último de retificação, no entanto, esses Estudantes não realizarão o Exame.

- Estudantes Concluintes dos Cursos de Bacharelado: Estudantes que tenham integralizado $80 \%$ ou mais da carga horária mínima do currículo do curso definido pelas IES e não tenham colado grau até o último dia do período de retificação de inscrições do Enade. A inscrição do Estudante habilitado para o Enade deverá ocorrer independentemente de haver registro de participação em edições anteriores.

A prova é composta de duas partes:

- Formação Geral (FG): composta de 10 questões, sendo 8 de múltipla escolha e 2 discursivas.

- Componentes Específicos (CE): composta de 30 questões, sendo 27 de múltipla escola e 3 discursivas.

\subsection{Questionário do Estudante}

É um instrumento do Sistema ENADE que tem como objetivo traçar o perfil socioeconômico dos estudantes e levantar informações sobre a construção de seus processos formativos, que são elementos importantes para a compreensão dos resultados dos estudantes concluintes inscritos no ENADE, e de suporte nos processos de avaliação de cursos de graduação e instituições de ensino superior.

Os estudantes inscritos devem realizar o preenchimento completo exclusivamente no Sistema ENADE como forma decisiva para participação no exame. Serve como objeto de verificação no processo de atribuição de sua regularidade no exame como também, requisito necessário para identificação do local da prova. 
O INEP realiza a agregação das respostas para cada curso de graduação com total sigilo da identificação dos estudantes.

\section{METODOLOGIA}

Os dados utilizados neste estudo foram coletados a partir dos microdados disponibilizados pelo INEP, relativos ao ENADE realizado nos anos de 2014 (INEP, 2016) e 2017 (INEP, 2018) para o curso de Engenharia de Produção código 6208, de acordo com código da área de enquadramento do curso no ENADE. Os microdados do INEP se constituem no menor nível de desagregação de dados recolhidos por pesquisas, avaliações e exames realizados.

Para medir a percepção dos alunos quanto ao desempenho das IES's foram analisadas as respostas de 42 questões obtidas do Questionário do Estudante, respondidas por cada participante do ENADE. Tais questões refletem perguntas relacionadas aos aspectos relacionados ao desempenho das instituições.

As questões selecionadas foram as de código QE_I27 até a QE_I68, sendo classificadas em quatro categorias: i) processo ensino-aprendizagem; ii) gestão educacional; iii) ação docente; iv) estrutura física. A distribuição das mesmas é apresentada no quadro 1. Para conhecer o conteúdo de cada questão, basta acessar http://portal.inep.gov.br/questionario-doestudante.

Quadro 1 - Distribuição das questões avaliadas para o desempenho institucional.

\begin{tabular}{|c|l|}
\hline CATEGORIAS & \multicolumn{1}{c|}{ QUESTÕES AVALIADAS } \\
\hline $\begin{array}{c}\text { Processo Ensino- } \\
\text { Aprendizagem }\end{array}$ & $\begin{array}{l}\text { QE_I27; QE_I28; QE_I29; QE_I30; QE_I31; QE_I32; QE_I33; QE_I34; QE_I35; } \\
\text { QE_I36; QE_I51; QE_I55; QE_I66 }\end{array}$ \\
\hline \multirow{2}{*}{$\begin{array}{c}\text { Gestão Educacional } \\
\text { Ação Docente }\end{array}$} & $\begin{array}{l}\text { QE_I40; QE_I41; QE_I42; QE_I43; QE_I44; QE_I45; QE_I46; QE_I47; QE_I48; } \\
\text { QE_I49; QE_I50; QE_I52; QE_I53; QE_I54; QE_I59; QE_I60; QE_I67 }\end{array}$ \\
\hline QE_trutura Física & QE_I61; QE_I62; QE_I63; QE_I64; QE_I65; QE_I68 \\
\hline
\end{tabular}

Fonte: Os autores

As respostas variam de 1 a 8 , onde 1 significa "discordo totalmente" e 6 "concordo totalmente", 2 a 5 representam uma escala intermediária de concordância, 7 representa "não sei responder" e 8 "não se aplica". Na análise estas duas últimas não serão consideradas.

Para que seja possível atribuir uma pontuação numérica a cada questão, as questões de 1 a 6 serão associadas a uma escala Likert e receberão pesos específicos conforme mostrado a seguir:

a) Resposta 1 - peso 1,5

b) Resposta 2 - peso 3,0

c) Resposta 3 - peso 4,5

d) Resposta 4 - peso 6,0

e) Resposta 5 - peso 8,0

f) Resposta 6 - peso 10,0

A pontuação de cada questão será calculada segundo a equação 1: 
(C) COBENCE

"Os desafios para formar hoje o engenheiro do amanhã"
$\mathrm{O}$ a $\mathrm{O3}$ de dezembro Evento On-line

$$
\text { Pont_ } \mathrm{QE}_{\mathrm{i}}=\mathrm{PC}_{1} * \mathrm{PS}_{1}+\mathrm{PC}_{2} * \mathrm{PS}_{2}+\mathrm{PC}_{3} * \mathrm{PS}_{3}+\mathrm{PC}_{4} * \mathrm{PS}_{4}+\mathrm{PC}_{5} * \mathrm{PS}_{5}+\mathrm{PC}_{6} * \mathrm{PS}_{6}
$$

Onde:

- Pont_QE $\mathrm{QE}_{\mathrm{i}}$ - Pontuação da iésima questão;

- $\mathrm{PC}_{1} \mathrm{a} \mathrm{PC}_{6}$ - Percentual de cada resposta (de 1 a 6);

- $\mathrm{PS}_{1}$ a $\mathrm{PS}_{6}$ - Pesos atribuídos às respostas de 1 a 6 ;

\section{RESULTADOS E DISCUSSÕES}

Antes de proceder com o cálculo da pontuação das questões selecionadas, executou-se primeiramente algumas estatísticas descritivas para melhor entendimento dos dados relacionados às Instituições de Ensino Superior. As mesmas estão descritas nos gráficos 1, 2 e 3.

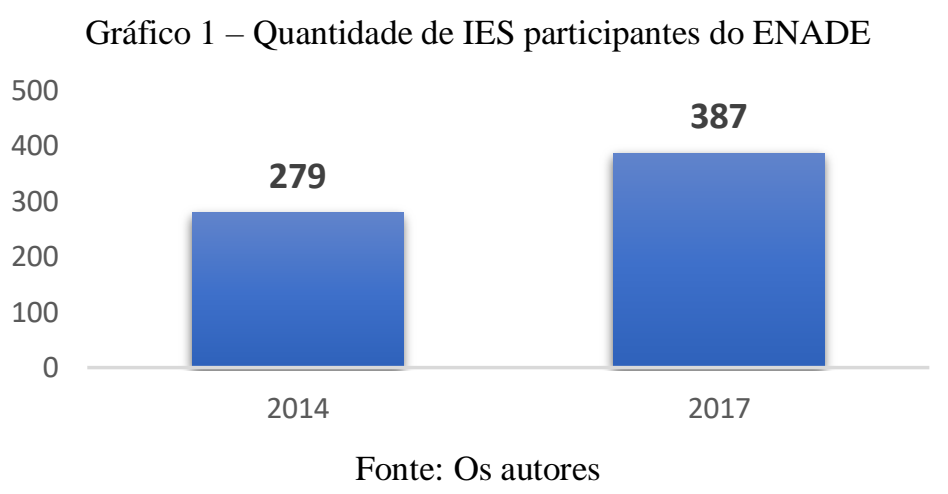

Gráfico 2 - Quantidade de IES por Categoria

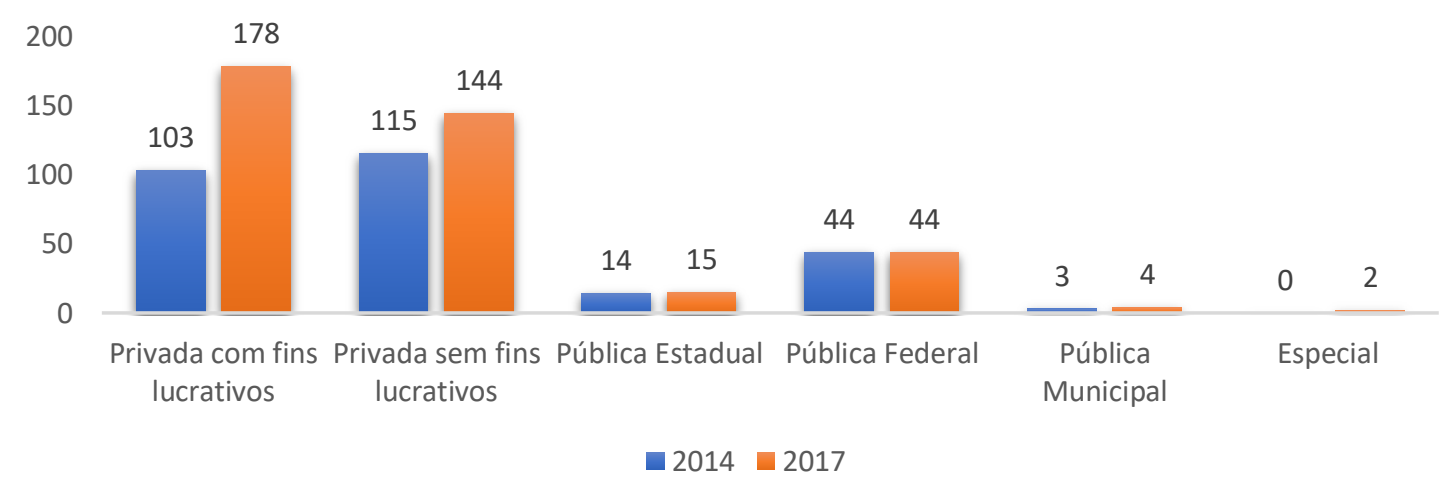

Fonte: Os autores

Gráfico 3 - Quantidade de estudantes por categoria de IES

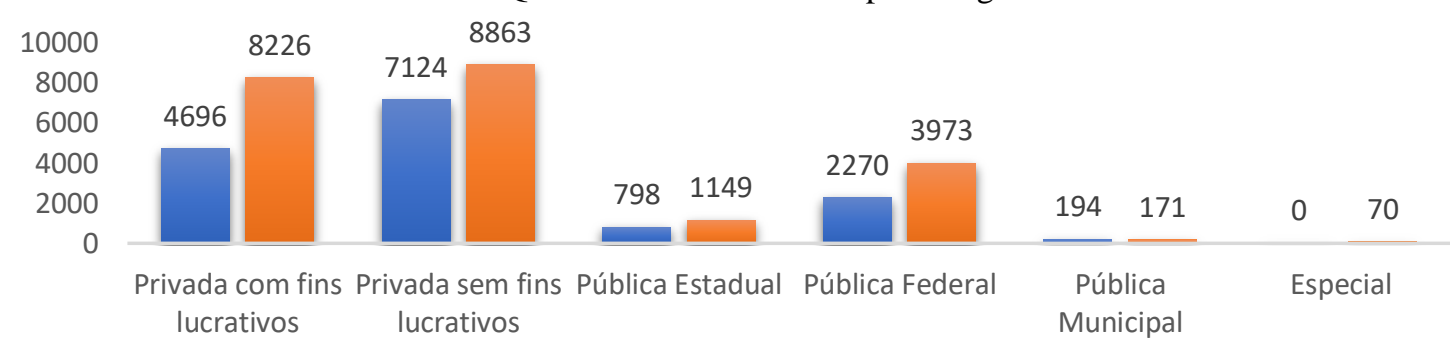

- $2014 \square 2017$

Fonte: Os autores 


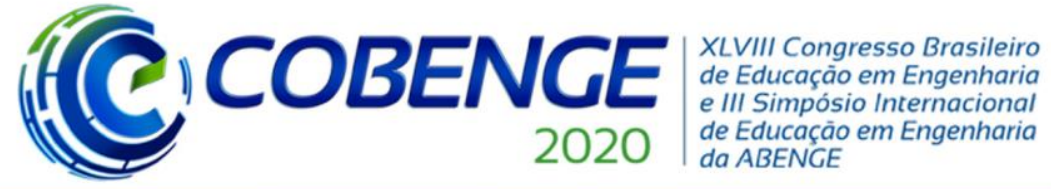

"Os desafios para formar hoje o engenheiro do amanhã"
$\mathrm{Ol}$ a $\mathrm{O3}$ de dezembro Evento On-line

Observando os gráficos 1 e 2, verifica-se um aumento de 108 IES participantes do ENADE de 2014 para 2017, representando um percentual de 39\%. Esta diferença foi influenciada em sua quase totalidade pelo aumento das instituições privadas. Porém, analisando o gráfico 3, verifica-se que o aumento da quantidade de participantes no ENADE não se deve somente às instituições privadas. Mesmo mantendo a quantidade de IES públicas, a quantidade de estudantes destas aumentou em 44\% nas estaduais e 75\% nas federais. Somente as públicas municipais apresentaram uma redução na quantidade de participantes, porém, pouco representativa no total.

O cálculo da pontuação de cada questão foi realizado considerando um total de 15.082 respostas no ano de 2014 e 22.452 respostas para 2017, onde foram excluídos os dados referentes às respostas 7 e 8 e vazias. No quadro 2 são apresentados os resultados.

Quadro 2 - Cálculo da pontuação de cada questão avaliada.

\begin{tabular}{|c|c|c|c|c|c|c|c|c|c|c|c|c|c|c|}
\hline \multirow{3}{*}{ QUESTÃO } & \multicolumn{7}{|c|}{2014} & \multicolumn{7}{|c|}{2017} \\
\hline & 1,5 & 3 & 4,5 & 6 & 8 & 10 & \multirow{2}{*}{ Pontuação } & 1,5 & 3 & 4,5 & 6 & 8 & 10 & \multirow{2}{*}{ Pontuação } \\
\hline & 1 & 2 & 3 & 4 & 5 & 6 & & 1 & 2 & 3 & 4 & 5 & 6 & \\
\hline QE_I27 & $0,4 \%$ & $0,9 \%$ & $3,4 \%$ & $14,0 \%$ & $30,0 \%$ & $51,3 \%$ & 8,56 & $0,5 \%$ & $1,3 \%$ & $4,4 \%$ & $14,2 \%$ & $29,7 \%$ & $50,0 \%$ & 8,47 \\
\hline QE_I28 & $0,6 \%$ & $1,5 \%$ & $4,2 \%$ & $13,9 \%$ & $29,1 \%$ & $50,8 \%$ & 8,48 & $1,1 \%$ & $2,2 \%$ & $5,5 \%$ & $14,2 \%$ & $28,5 \%$ & $48,6 \%$ & 8,31 \\
\hline QE_I29 & $1,1 \%$ & $3,1 \%$ & $6,8 \%$ & $17,8 \%$ & $29,1 \%$ & $42,1 \%$ & 8,02 & $1,6 \%$ & $3,5 \%$ & $8,1 \%$ & $18,1 \%$ & $27,7 \%$ & $41,1 \%$ & 7,90 \\
\hline QE_I30 & $1,6 \%$ & $3,8 \%$ & $7,5 \%$ & $17,8 \%$ & $27,0 \%$ & $42,5 \%$ & 7,94 & $2,3 \%$ & $4,4 \%$ & $9,0 \%$ & $17,6 \%$ & $25,8 \%$ & $40,8 \%$ & 7,78 \\
\hline QE_I31 & $0,7 \%$ & $2,0 \%$ & $4,6 \%$ & $12,6 \%$ & $28,2 \%$ & $51,9 \%$ & 8,48 & $1,1 \%$ & $2,1 \%$ & $5,1 \%$ & $13,1 \%$ & $27,2 \%$ & $51,6 \%$ & 8,42 \\
\hline QE_I32 & $0,4 \%$ & $1,2 \%$ & $3,3 \%$ & $9,5 \%$ & $25,1 \%$ & $60,6 \%$ & 8,82 & $0,6 \%$ & $1,2 \%$ & $3,3 \%$ & $9,2 \%$ & $23,7 \%$ & $62,0 \%$ & 8,84 \\
\hline QE_I33 & $0,5 \%$ & $1,3 \%$ & $3,2 \%$ & $9,7 \%$ & $27,8 \%$ & $57,6 \%$ & 8,75 & $0,7 \%$ & $1,3 \%$ & $3,8 \%$ & $10,7 \%$ & $28,4 \%$ & $55,2 \%$ & 8,65 \\
\hline QE_I34 & $0,6 \%$ & $1,4 \%$ & $4,1 \%$ & $11,9 \%$ & $28,3 \%$ & $53,8 \%$ & 8,58 & $0,9 \%$ & $1,7 \%$ & $4,6 \%$ & $12,3 \%$ & $28,3 \%$ & $52,3 \%$ & 8,50 \\
\hline QE_I35 & $0,6 \%$ & $1,6 \%$ & $4,0 \%$ & $12,2 \%$ & $28,8 \%$ & $52,8 \%$ & 8,55 & $0,9 \%$ & $1,6 \%$ & $4,4 \%$ & $12,8 \%$ & $28,7 \%$ & $51,6 \%$ & 8,48 \\
\hline QE_I36 & $0,4 \%$ & $1,2 \%$ & $3,6 \%$ & $13,5 \%$ & $32,5 \%$ & $48,9 \%$ & 8,49 & $0,8 \%$ & $1,6 \%$ & $4,4 \%$ & $13,6 \%$ & $30,4 \%$ & $49,3 \%$ & 8,43 \\
\hline QE_I37 & $2,2 \%$ & $3,6 \%$ & $7,6 \%$ & $19,3 \%$ & $29,2 \%$ & $38,1 \%$ & 7,79 & $2,9 \%$ & $4,4 \%$ & $8,7 \%$ & $17,9 \%$ & $26,8 \%$ & $39,2 \%$ & 7,71 \\
\hline QE_I38 & $1,1 \%$ & $3,0 \%$ & $7,5 \%$ & $20,3 \%$ & $30,7 \%$ & $37,4 \%$ & 7,86 & $1,7 \%$ & $3,4 \%$ & $8,4 \%$ & $19,6 \%$ & $29,0 \%$ & $37,9 \%$ & 7,79 \\
\hline QE_I39 & $0,8 \%$ & $2,5 \%$ & $6,2 \%$ & $16,9 \%$ & $29,9 \%$ & $43,8 \%$ & 8,15 & $1,2 \%$ & $2,9 \%$ & $7,3 \%$ & $16,4 \%$ & $29,4 \%$ & $42,8 \%$ & 8,05 \\
\hline QE_I40 & $3,4 \%$ & $4,6 \%$ & $9,7 \%$ & $20,8 \%$ & $27,4 \%$ & $34,2 \%$ & 7,48 & $3,7 \%$ & $5,1 \%$ & $10,6 \%$ & $19,2 \%$ & $25,1 \%$ & $36,3 \%$ & 7,48 \\
\hline QE_I41 & $3,9 \%$ & $5,7 \%$ & $8,6 \%$ & $15,8 \%$ & $23,6 \%$ & $42,4 \%$ & 7,69 & $4,1 \%$ & $5,0 \%$ & $8,4 \%$ & $14,7 \%$ & $22,5 \%$ & $45,4 \%$ & 7,81 \\
\hline QE_I42 & $0,7 \%$ & $1,7 \%$ & $4,4 \%$ & $12,6 \%$ & $28,5 \%$ & $52,2 \%$ & 8,51 & $0,8 \%$ & $1,5 \%$ & $4,3 \%$ & $12,1 \%$ & $27,2 \%$ & $54,1 \%$ & 8,56 \\
\hline QE_I43 & $3,9 \%$ & $5,0 \%$ & $8,2 \%$ & $16,4 \%$ & $24,4 \%$ & $42,1 \%$ & 7,73 & $4,3 \%$ & $4,8 \%$ & $8,5 \%$ & $14,3 \%$ & $22,9 \%$ & $45,2 \%$ & 7,80 \\
\hline QE_I44 & $4,5 \%$ & $5,5 \%$ & $8,9 \%$ & $16,7 \%$ & $23,6 \%$ & $40,8 \%$ & 7,60 & $4,9 \%$ & $5,3 \%$ & $8,6 \%$ & $15,5 \%$ & $22,5 \%$ & $43,2 \%$ & 7,67 \\
\hline QE_I45 & $2,7 \%$ & $4,7 \%$ & $8,7 \%$ & $16,5 \%$ & $25,3 \%$ & $42,1 \%$ & 7,80 & $2,7 \%$ & $4,3 \%$ & $8,8 \%$ & $15,6 \%$ & $23,8 \%$ & $44,8 \%$ & 7,88 \\
\hline QE_I46 & $6,8 \%$ & $7,7 \%$ & $11,2 \%$ & $19,4 \%$ & $21,0 \%$ & $33,9 \%$ & 7,07 & $7,0 \%$ & $6,3 \%$ & $10,8 \%$ & $16,8 \%$ & $21,0 \%$ & $38,2 \%$ & 7,28 \\
\hline QE_I47 & $2,4 \%$ & $4,9 \%$ & $8,9 \%$ & $18,7 \%$ & $28,2 \%$ & $36,9 \%$ & 7,65 & $3,5 \%$ & $5,8 \%$ & $10,7 \%$ & $18,2 \%$ & $24,7 \%$ & $37,1 \%$ & 7,49 \\
\hline QE_I48 & $4,6 \%$ & $7,2 \%$ & $11,1 \%$ & $20,9 \%$ & $24,7 \%$ & $31,5 \%$ & 7,17 & $5,7 \%$ & $7,6 \%$ & $11,9 \%$ & $18,9 \%$ & $23,3 \%$ & $32,6 \%$ & 7,11 \\
\hline QE_I49 & $0,9 \%$ & $2,6 \%$ & $6,0 \%$ & $16,6 \%$ & $31,2 \%$ & $42,8 \%$ & 8,13 & $1,3 \%$ & $2,6 \%$ & $7,1 \%$ & $16,4 \%$ & $29,4 \%$ & $43,1 \%$ & 8,07 \\
\hline QE_I50 & $1,7 \%$ & $2,1 \%$ & $3,7 \%$ & $11,5 \%$ & $23,9 \%$ & $57,2 \%$ & 8,57 & $2,3 \%$ & $2,0 \%$ & $4,5 \%$ & $10,0 \%$ & $22,8 \%$ & $58,4 \%$ & 8,56 \\
\hline QE_I51 & $0,9 \%$ & $1,4 \%$ & $3,0 \%$ & $10,6 \%$ & $25,9 \%$ & $58,3 \%$ & 8,72 & $1,4 \%$ & $1,6 \%$ & $3,8 \%$ & $10,2 \%$ & $25,9 \%$ & $57,1 \%$ & 8,64 \\
\hline QE_I52 & $12,3 \%$ & $7,8 \%$ & $9,4 \%$ & $14,9 \%$ & $19,0 \%$ & $36,6 \%$ & 6,92 & $13,6 \%$ & $7,1 \%$ & $9,8 \%$ & $14,5 \%$ & $18,0 \%$ & $37,1 \%$ & 6,88 \\
\hline QE_I53 & $16,9 \%$ & $8,3 \%$ & $8,5 \%$ & $12,9 \%$ & $17,2 \%$ & $36,2 \%$ & 6,65 & $17,3 \%$ & $7,4 \%$ & $8,8 \%$ & $12,8 \%$ & $16,4 \%$ & $37,3 \%$ & 6,69 \\
\hline QE_I54 & $3,0 \%$ & $3,6 \%$ & $5,8 \%$ & $12,6 \%$ & $21,7 \%$ & $53,3 \%$ & 8,24 & $2,6 \%$ & $3,1 \%$ & $6,0 \%$ & $11,4 \%$ & $21,3 \%$ & $55,6 \%$ & 8,35 \\
\hline QE_I55 & $1,0 \%$ & $2,4 \%$ & $5,4 \%$ & $16,0 \%$ & $31,3 \%$ & $43,9 \%$ & 8,19 & $1,1 \%$ & $2,1 \%$ & $6,1 \%$ & $15,8 \%$ & $30,3 \%$ & $44,6 \%$ & 8,19 \\
\hline QE_I56 & $2,2 \%$ & $4,7 \%$ & $7,6 \%$ & $17,1 \%$ & $27,2 \%$ & $41,3 \%$ & 7,84 & $2,0 \%$ & $3,4 \%$ & $7,7 \%$ & $16,3 \%$ & $26,9 \%$ & $43,8 \%$ & 7,98 \\
\hline QE_I57 & $0,7 \%$ & $2,3 \%$ & $6,0 \%$ & $16,3 \%$ & $32,4 \%$ & $42,4 \%$ & 8,15 & $0,6 \%$ & $1,9 \%$ & $6,0 \%$ & $15,8 \%$ & $31,7 \%$ & $44,0 \%$ & 8,22 \\
\hline QE_I58 & $0,7 \%$ & $1,9 \%$ & $4,3 \%$ & $11,2 \%$ & $25,1 \%$ & $56,9 \%$ & 8,63 & $1,0 \%$ & $2,2 \%$ & $5,2 \%$ & $12,7 \%$ & $24,9 \%$ & $54,1 \%$ & 8,47 \\
\hline QE_I59 & $2,8 \%$ & $4,1 \%$ & $6,6 \%$ & $14,5 \%$ & $26,4 \%$ & $45,7 \%$ & 8,01 & $3,1 \%$ & $3,8 \%$ & $7,4 \%$ & $13,9 \%$ & $24,4 \%$ & $47,4 \%$ & 8,02 \\
\hline QE_I60 & $4,2 \%$ & $5,8 \%$ & $8,7 \%$ & $16,8 \%$ & $24,1 \%$ & $40,4 \%$ & 7,61 & $4,0 \%$ & $4,6 \%$ & $8,6 \%$ & $15,5 \%$ & $23,9 \%$ & $43,4 \%$ & 7,77 \\
\hline QE_I61 & $2,6 \%$ & $4,3 \%$ & $7,4 \%$ & $15,2 \%$ & $26,9 \%$ & $43,7 \%$ & 7,93 & $2,2 \%$ & $3,8 \%$ & $7,2 \%$ & $15,1 \%$ & $25,8 \%$ & $45,9 \%$ & 8,03 \\
\hline QE_I62 & $4,1 \%$ & $5,6 \%$ & $8,3 \%$ & $16,2 \%$ & $25,4 \%$ & $40,3 \%$ & 7,64 & $3,7 \%$ & $4,9 \%$ & $8,4 \%$ & $15,6 \%$ & $24,7 \%$ & $42,7 \%$ & 7,76 \\
\hline QE_I63 & $4,0 \%$ & $5,7 \%$ & $8,6 \%$ & $16,8 \%$ & $25,5 \%$ & $39,5 \%$ & 7,61 & $3,5 \%$ & $4,7 \%$ & $8,6 \%$ & $15,7 \%$ & $24,9 \%$ & $42,6 \%$ & 7,77 \\
\hline QE_I64 & $1,2 \%$ & $3,1 \%$ & $5,4 \%$ & $13,4 \%$ & $26,2 \%$ & $50,8 \%$ & 8,33 & $1,1 \%$ & $2,1 \%$ & $5,3 \%$ & $11,8 \%$ & $26,2 \%$ & $53,6 \%$ & 8,48 \\
\hline QE_I65 & $5,9 \%$ & $5,2 \%$ & $6,7 \%$ & $13,3 \%$ & $22,2 \%$ & $46,7 \%$ & 7,79 & $4,8 \%$ & $3,5 \%$ & $6,0 \%$ & $11,4 \%$ & $21,4 \%$ & $52,9 \%$ & 8,14 \\
\hline QE_I66 & $1,3 \%$ & $2,8 \%$ & $6,1 \%$ & $15,0 \%$ & $28,4 \%$ & $46,5 \%$ & 8,20 & $1,6 \%$ & $2,6 \%$ & $6,3 \%$ & $14,0 \%$ & $26,5 \%$ & $49,1 \%$ & 8,25 \\
\hline QE_I67 & $5,7 \%$ & $7,5 \%$ & $10,0 \%$ & $17,8 \%$ & $21,9 \%$ & $37,2 \%$ & 7,29 & $5,1 \%$ & $5,6 \%$ & $9,2 \%$ & $15,8 \%$ & $22,5 \%$ & $41,8 \%$ & 7,59 \\
\hline QE_I68 & $3,4 \%$ & $5,0 \%$ & $7,0 \%$ & $12,8 \%$ & $22,9 \%$ & $49,0 \%$ & 8,01 & $2,8 \%$ & $4,0 \%$ & $6,9 \%$ & $12,0 \%$ & $21,5 \%$ & $52,9 \%$ & 8,20 \\
\hline \multicolumn{7}{|c|}{ PONTUAÇÃO MÉDIA 2014} & 7,99 & \multicolumn{6}{|c|}{ PONTUAÇÃO MÉDIA 2017} & 8,01 \\
\hline \multicolumn{7}{|c|}{ DESVIO PADRÃO } & $\mathbf{0 , 5 2}$ & \multicolumn{6}{|c|}{ DESVIO PADRÃO } & 0,49 \\
\hline
\end{tabular}

Fonte: Os autores 
A partir dos dados apresentados no quadro 2 obtemos a pontuação média geral e o desvio padrão em cada ano. Em 2014, o valor foi de 7,99 pontos, com desvio padrão de 0,52, e em $2017,8,01$ pontos com desvio padrão de 0,49 . Tal resultado demonstra que não houve alteração na percepção dos estudantes quanto ao desempenho das instituições de ensino superior, assim como a variabilidade reduziu levemente em 0,03 pontos.

Os resultados referentes a cada categoria estão demonstrados no gráfico 4. Eles mostram uma redução de 0,07 pontos de 2014 para 2017 na categoria Processo Ensino-Aprendizagem; um aumento de 0,05 pontos na categoria Gestão Educacional; uma redução de 0,03 pontos na categoria Ação Docente; e um aumento de 0,18 pontos em Estrutura Física.

Gráfico 4 - Pontuações médias por categoria de avaliação das IES's

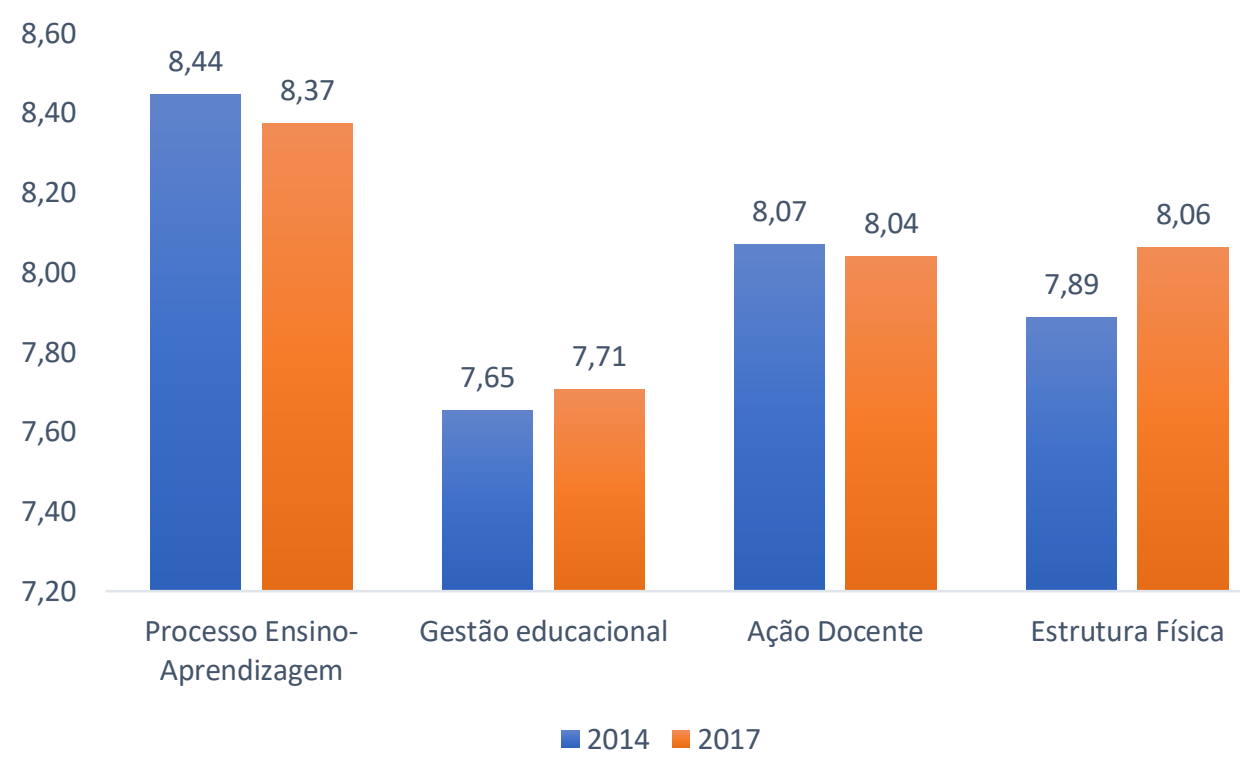

Fonte: Os autores

Apesar da pequena variação entre as categorias, algumas considerações devem ser realizadas:

- Na categoria Processo Ensino-Aprendizagem houve regressão em 11 das 13 questões avaliadas. Onde os maiores valores negativos referem-se às questões QE_I30 (o curso propiciou experiências de aprendizagem inovadoras) com -2,08\% e QE_I28 (os conteúdos abordados nas disciplinas do curso favoreceram sua atuação em estágios ou em atividades de iniciação profissional) com -1,93\%;

- Na categoria Gestão Educacional, as questões que apresentaram maiores evoluções foram a QE_I67 (a instituição promoveu atividades de cultura, de lazer e de interação social) com 4,04\% e QE_I46 (a instituição ofereceu oportunidades para os estudantes atuarem como representantes em órgãos colegiados) com 2,91\%. Enquanto que a questão que apresentou maior redução foi a QE_I47 (o curso favoreceu a articulação do conhecimento teórico com atividades práticas) com -2,14\%;

- Na categoria Ação Docente, houve evolução em duas das seis questões avaliadas. Estas foram a QE_I56 (os professores apresentaram disponibilidade para atender os estudantes fora do horário das aulas) com 1,82\% e QE_I57 (os professores demonstraram domínio dos conteúdos abordados nas disciplinas) com 0,84\%. Das questões que tiveram redução, destaca-se a questão QE_I58 (os professores utilizaram tecnologias da informação e comunicação (TICs) como estratégia de ensino (projetor 
multimídia, laboratório de informática, ambiente virtual de aprendizagem)) com $1,75 \%$;

- A categoria Estrutura Física foi aquela que apresentou maior evolução entre os anos, havendo aumento em todas as questões. Destaca-se a questão QE_I65 (a instituição contou com biblioteca virtual ou conferiu acesso a obras disponíveis em acervos virtuais) com 4,45\% e a questão QE_I68 (a instituição dispôs de refeitório, cantina e banheiros em condições adequadas que atenderam as necessidades dos seus usuários) com aumento de $2,34 \%$.

\section{CONSIDERAÇÕES FINAIS}

O presente trabalho analisou a percepção dos estudantes de Engenharia de Produção, participantes do ENADE nos anos de 2014 e 2017, a partir das respostas de 42 questões qualitativas pertencentes ao Questionário do Estudante que representam aspectos da qualidade dos serviços das Instituições de Ensino Superior, quantificando os percentuais de cada tipo de resposta a partir de valores de uma escala Likert, gerando uma pontuação para cada questão.

A partir disso, verificou-se que não houve variação na pontuação média geral entre 2014 e 2017, demonstrando que a percepção dos estudantes não mudou entre estes anos. Além disso, foi evidenciado um aumento da pontuação relacionado às categorias de Gestão Educacional e de Estrutura Física, ao contrário das categorias de Processo de ensinoaprendizagem e das ações por parte do corpo docente que, na avaliação dos estudantes, precisam reduziu entre os anos.

Esse panorama apresentado deve ser analisado e discutido por toda a comunidade acadêmica e sociedade, pois seus resultados e impressões refletem diretamente no desempenho dos estudantes no ENADE, e por consequência, em sua vida profissional.

Os resultados apresentados nesse estudo mostram um aumento na quantidade de IES participantes no ENADE entre os anos de 2014 e 2017, tendo como destaque nesse aumento, o número expressivo de instituições privadas como também, de estudantes ingressantes nessa categoria de IES. As instituições públicas apesar de estar presente em menor número, ainda conseguem ser bem atrativas ao ingresso de estudantes.

A estratificação dos dados por categoria de IES tende a reforçar a influência das instituições privadas sobre os resultados de cada categoria. Pois ações ligadas à Gestão educacional e principalmente, à Estrutura Física são "menos burocráticas" de realização nas instituições privadas do que nas instituições públicas frente às legislações cabíveis a ambas, ficando o "impacto" dessas ações nas instituições privadas a depender do aporte de capital para investimento.

É possível classificar essas ações como "extracurriculares", pois não determinam de forma direta a formação do perfil profissional do estudante, mas são de extrema importância na condução desse processo. No entanto, as ações relacionadas às categorias do processo de ensino-aprendizagem e da ação docente estão diretamente relacionadas à formação do currículo profissional do estudante que estão ligadas a paradigmas e práticas pedagógicas. A evolução dessas últimas acontece de forma mais lenta e gradual, e dependem da voluntariedade e do aporte de conhecimento empregado, mas que na percepção dos estudantes em qualquer categoria de IES precisam evoluir. Por fatores de competitividade e sobrevivência no mercado, as instituições privadas necessitam encontrar alternativas e elaborar métodos que melhorem a cada dia a qualidade dessas ações como forma de sempre adquirir a preferência do estudante.

A continuidade dessa análise incluindo dados das próximas edições do ENADE, associada a estudos e relatos de trabalho vivenciados tanto por instituições privadas como públicas diante dos aspectos apresentados nas quatro categorias abordadas nesse estudo são 
recomendações para trabalhos futuros como forma de melhor diagnosticar as causas e possíveis soluções afim de promover a melhoria contínua do desempenho dos estudantes de Engenharia de Produção no referido exame.

\section{REFERÊNCIAS}

BITTENCOURT, H. R.; VIALI, L.; BELTRAME, E. A engenharia de produção no Brasil: um panorama dos cursos de graduação e pós-graduação. Revista de Ensino de Engenharia, Brasília, v. 29, n. 1, p. 11-19, 2010.

CAMELO, M.R.; MARCIÃO, M.I.R. Gestão da Qualidade aplicada ao plano pedagógico do curso institucional de Instituições de Ensino Superior. Anais do V Congresso Nacional de Educação, 2019.

CONRADSEN, N.; LYSTLUND, M. The vision of next generation manufacturing: how a company can start. Integrated Manufacturing Systems, v.14, n.4, p. 324-333, 2003.

INEP - Instituto Nacional de Pesquisas Educacionais Anísio Teixeira. Dados abertos Microdados - ENADE 2014, 2016. Disponível em:

< http://download.inep.gov.br/microdados/Enade_Microdados/microdados_enade_2014.zip>. Acesso em 02/04/2020.

INEP - Instituto Nacional de Pesquisas Educacionais Anísio Teixeira. Dados abertos Microdados - ENADE 2017, 2018. Disponível em:

$<$ http://download.inep.gov.br/microdados/Enade_Microdados/microdados_Enade_2017_port al_2018.10.09.zip>. Acesso em 02/04/2020.

INEP - Instituto Nacional de Pesquisas Educacionais Anísio Teixeira. Censo da Educação do Ensino Superior - Notas Estatísticas, 2019. Disponível em:

<http://download.inep.gov.br/educacao_superior/censo_superior/documentos/2019/censo_da_ educacao_superior_2018-notas_estatisticas.pdf>. Acesso em: 30 abr. 2020.

INEP - Instituto Nacional de Pesquisas Educacionais Anísio Teixeira. Sinopses Estatísticas da Educação Superior - Graduação, 2020. Disponível em:

<http://portal.inep.gov.br/web/guest/sinopses-estatisticas-daeducacao-superior>. Acesso em: 20 abr. 2020.

OLIVEIRA, V. F. de; ALMEIDA, N. N. de; CARVALHO, D. M. DE; PEREIRA, F. A. A. Um estudo sobre a expansão da formação em engenharia no Brasil. Revista de Ensino de Engenharia, Brasília, v. 32, n. 3, 2013.

OLIVEIRA, Vanderli Fava; FERNANDES, Pedro H. P (2015). Impactos da Reorganização dos Cursos de Engenharia para o ENADE 2014. Revista Eletrônica Engenharia Viva. v.1, p.83- 96, 2015.

QUEIROZ, F. C. B. P.; QUEIROZ, J. V.; HEKIS, H. R.; PEREIRA, F. A. B.; SILVA, B. C. L. C. Qualidade nas instituições de ensino superior (IES): diferenças entre conceitos e 
metodologias de aferição. 2011. Encontro Nacional de Engenharia e Gestão Industrial (ENEGI 2011). Guimarães, Portugal.

RIBEIRO, I. S.; ANJOS, E. S.; BARBOSA, A. S.; SANTANA, A. M. O. Avaliação dos cursos de Engenharia de Produção das Universidades Federais do Nordeste baseado no método multicritério PROMETHEE. In: Anais do XXXIX Encontro Nacional de Engenharia de Produção, 2019.

\title{
AN ANALYSIS OF THE PERCEPTION OF PRODUCTION ENGINEERING STUDENTS AS TO THE QUALITY OF HIGHER EDUCATION INSTITUTIONS IN 2014 AND 2017
}

\begin{abstract}
The number of courses in production engineering as well as new students has been registering a frequent increase. In this sense, this study analyzed the impact of actions by Higher Education Institutions represented by qualitative variables. For this, the perception of students of Production Engineering courses regarding the quality of Higher Education Institutions in the years 2014 and 2017 was used as an indicator, analyzing the answers to 42 qualitative questions pertaining to the Student Questionnaire. Such questions reflect questions related to aspects of the institutions' performance. From the analysis of the results, it was found that there is no significant difference in the average general perception of students. The study demonstrated the relevance of statistical analysis on the results of ENADE, and directs that new variables that may influence these results be analyzed in future articles.
\end{abstract}

Keywords: Production engineering; Enade; Student questionnaire; Perception of the student. 